\title{
Metahemoglobinemia secundaria a inhalación de "poppers": caso clínico y revisión de la literatura
}

\section{Methemoglobinemia secondary to amyl nitrite inhalation. A case report and literature review}

\author{
Tamara Muñoz ${ }^{1}$, Arturo Peña² ${ }^{2}$ Oscar Corsi ${ }^{3}$
}

\section{Resumen}

El nitrito de amilo, conocida como "poppers", se ha masificado como droga recreacional en parte por sus efectos con objetivos sexuales. Su consumo se asocia a complicaciones psiquiátricas y médicas. Reportamos el caso de un paciente que se presenta con metahemoglobinemia moderada secundaria a la inhalación de nitrito de amilo asociado a alcohol. Al ingreso presenta cianosis peribucal y en extremidades, disociación entre oximetría de pulso y presión parcial de oxígeno en gases arteriales, además de metahemoglobinemia $29,9 \%$. Se descartan otras intoxicaciones y causas primarias de metahemoglobinemia. Se maneja con oxigenoterapia, hidratación y ácido ascórbico, presentando una evolución favorable. Presentamos el primer caso en Chile de metahemoglobinemia secundaria al consumo de nitrito de amilo con fines recreativos.

Palabras clave: Metahemoglobinemia; Nitrato de amilo; virus inmunodeficiencia humana (VIH)

\begin{abstract}
Amyl nitrite, known as "poppers", has become popular as a recreational drug for sexual purposes. Its consumption is associated with psychiatric and medical complications. We report the case of a patient presenting with moderate methemoglobinemia secondary to amyl nitrite inhalation associated with alcohol. At admission, perioral and extremities cyanosis, a dissociation between pulse oximetry and partial pressure of oxygen in arterial gases, and methemoglobinemia $29.9 \%$ were present. Other intoxications and primary causes of methemoglobinemia were ruled out. The patient receives oxygen therapy, hydration, and ascorbic acid, presenting a favorable evolution. We report the first Chilean's case of methemoglobinemia secondary to amyl nitrite consumption for recreational purposes.
\end{abstract}

Keywords: Methemoglobinemia; Amyl Nitrite; HIV-1

Fecha de envío: 31 de Dicembre de 2019 - Fecha de aceptación: 11 de junio de 2020

\section{Introducción}

El nitrito de amilo es una sustancia altamente volátil descubierta en el siglo XIX, la cual fue usada originalmente para el manejo de la angina gracias a su efecto vasodilatador. En las últimas décadas, ha aumentado su uso como droga recreacional bajo el nombre de "poppers", particularmente con objetivos sexuales (Romanelli et al., 2004; Hunter et al., 2011). Su consumo al margen de una indicación médica se ha asociado a la ocurrencia de patologías psiquiátricas y médicas (Wu \& Ringwalt, 2006; Hunter et al., 2011; Luis et al., 2016). Dentro de estas, se han descrito casos de metahemoglobinemia de variable severidad (Hunter et al., 2011).
A continuación, reportamos el caso de un paciente que se presenta con metahemoglobinemia moderada secundaria al consumo de nitrito de amilo para describir un cuadro clínico que, en el futuro, podría ser habitual en los servicios de urgencia de país dada la creciente popularidad en el uso recreacional de esta sustancia.

\section{Caso clínico}

Presentamos el caso de un paciente masculino de 33 años con antecedente de infección por el virus de inmunodeficiencia humana $(\mathrm{VIH})$ con carga viral indetectable los últimos dos años, quien cambió su tratamiento antirretroviral por emtricitabina/tenofovir/

(1) Departamento de Otorrinolaringología, Facultad de Medicina, Universidad de Chile, Santiago, Chile

(2) Sección Medicina de Urgencia, Facultad de Medicina, Pontificia Universidad Católica de Chile, Santiago, Chile

(3) Departamento Medicina Interna, Facultad de Medicina, Pontificia Universidad Católica de Chile, Santiago, Chile

Autor de correspondencia ofcorsi@uc.cl 
efavirenz (formulación combinada) seis meses previos al ingreso, sin uso de cotrimoxazol ni dapsona. Él consulta en el servicio de urgencia del Hospital Clínico de la Red de Salud UC-Christus por un cuadro de dos horas de evolución caracterizado por cianosis peribucal y distal asociado a fatigabilidad, letargia, parestesias de extremidades inferiores y visión borrosa; él niega angina, palpitaciones o compromiso de conciencia. A la anamnesis dirigida refiere inhalación de nitrito de amilo en más de 10 oportunidades una hora previa al inicio del cuadro asociado a consumo de alcohol en moderada cantidad, en contexto recreativo, sin intención suicida.

Ingresa vigil, orientado, hemodinámicamente estable, PA 124/81 $\mathrm{mmHg}$, FC 97x`, FR 20x`y desaturación hasta $80 \%$ al oxímetro de pulso que revierte con administración de oxígeno con $\mathrm{FIO} 2$ 35\%. Al examen físico solo destaca cianosis peribucal y en extremidades, sin hallazgos cardiopulmonares. De los exámenes destaca metahemoglobina en $29,9 \%$ y ECG sin alteraciones; resto del laboratorio sanguíneo es detallado en la Tabla 1.

Se decide hospitalizar en el servicio de Intermedio Médico para monitorización y manejo de metahemoglobinemia secundaria a nitrito de amilo. Recibe oxigenoterapia, hidratación y ácido ascórbico oral.

Considerando la baja probabilidad de causas alternativas de metahemoglobinemia, se complementa estudio solo con glucosa-6-fosfato deshidrogenasa que muestra tiempo de decoloración del azul de metileno de 17 minutos (VN 10-20 minutos) y estudio de intoxicación por drogas por Inmunoensayo automatizado / Cromatografía gaseosa con espectrometría de masa (Toxilab ${ }^{\circledR}$, Roche) que solo detecta cafeína y acetaminofeno.

Presenta una favorable respuesta clínica y de laboratorio, disminuyendo metahemoglobina hasta $1,4 \%$ a las 12 horas desde el ingreso, sin dificultad respiratoria, hemodinámicamente estable, sin arritmias. De esta forma, se decide alta con los diagnósticos de Insuficiencia respiratoria aguda parcial secundaria a intoxicación por nitrito de amilo y metahemoglobinemia moderada secundaria, ambas recuperadas. Se deriva a sus controles médicos habituales.

\section{Discusión}

El nitrito de amilo, conocido comúnmente como "poppers", es una sustancia vasodilatadora altamente volátil. Desde su descubrimiento a fines del siglo XIX fue utilizado para el manejo de angina cardiaca gracias a su efecto vasodilatador, hasta su reemplazo por los nitratos en la década del sesenta (Romanelli et al., 2004; Hunter et al., 2011). Actualmente su uso se reserva como alternativa para el manejo de la intoxicación por cianuro (salvo aquellos casos con intoxicación por monóxido de carbono concomitante).
En las últimas décadas, su uso recreacional se ha popularizado en población joven por su efecto estimulante (Wu et al., 2005). La población gay, bisexual y hombres que tienen sexo con hombres concentra el consumo de nitrato de amilo, motivados por su efecto relajador del esfínter anal (Vaccher et al., 2020).

Ha sido descrita una mayor prevalencia de virus de inmunodeficiencia adquirida en la población consumidora de poppers, probablemente secundario a un mayor riesgo de relaciones sexuales sin protección (Li et al., 2014). Sin embargo, esta asociación no ha sido demostrada categóricamente (Romanelli et al., 2004; Wu et al., 2005). Dentro de la población infectada por VIH, el consumo de "poppers" se asocia a la identificación gay o bisexual y la participación en comercio sexual (Pepper et al., 2019).

En Chile no existen estudios específicos respecto al consumo de esta droga. Cifras oficiales publicadas muestran que las drogas "inhalables" - que incluyen el nitrito de amilo - muestran una baja prevalencia de consumo. En la población general, la mayor prevalencia de consumo alguna vez en la vida fue entre los años 2002 y 2008 (2,2 a 1,9\%), tras lo cual presentó una caída y posterior ascenso desde el 2012 al 2016 (1 a 1,3\%) (SENDA, 2016). En la población escolar, esta prevalencia de vida para el año 2017 alcanzó un $8,3 \%$-luego del peak de $10.3 \%$ el año 2015 - con una edad de inicio de consumo a los 13,1 años (SENDA, 2017).

El uso recreacional de nitrito de amilo no es inocuo. Se ha asociado con tendencia a conductas antisociales, criminales y síntomas depresivos (Wu \& Ringwalt, 2006). Por otro lado, el consumo de nitritos volátiles se asocia a síntomas como palpitaciones, mareos, náuseas y síncope (Hunter et al., 2011), y se ha descrito retinopatía/ maculopatía secundaria a su consumo (Luis et al., 2016). A nivel internacional se han publicado cerca de 30 casos de metahemoglobinemia secundaria al abuso de esta sustancia, la mayoría de los cuales con resultados favorables y sin consecuencias relevantes; pero también han sido resportado algunos casos fatales (Malhotra \& Hughes, 1996; Pajarón et al., 2003; Wilkerson, 2010; Hunter et al., 2011; Al-Lawati \& Murch, 2012; McCabe et al., 2012; Bernasconi et al., 2014).

La metahemoglobina se produce cuando el fierro del grupo heme de la hemoglobina pierde un electrón, pasando de su estado ferroso a un estado férrico. La metahemoglobina presenta una menor capacidad trasportadora de oxígeno, hecho que se suma al aumento de la afinidad de la hemoglobina normal por el oxígeno (Price, 2011). De este modo, ocurre un desplazamiento a la izquierda de la curva de disociación hemoglobina- $\mathrm{O}_{2}$ con la consecuente disminución de la entrega tisular. 
En condiciones normales, existe una pequeña concentración de metahemoglobina entre 0,5 y $3 \%$ la cual se mantiene estable gracias a los mecanismos de reducción intrínsecos, principalmente la vía del NADH catalizada por el citocromo b5 reductasa. Además, se describe la vía del NADPH la cual no tiene un rol en condiciones habituales, pero sí implicancias terapéuticas.

La metahemoglobinemia puede ser de origen congénito por a) defectos enzimáticos en aquellas participantes de la reducción de la hemoglobina (citocromo b5 reductasa) ó b) variaciones en la proteína hemoglobina (hemoglobina M). La metahemoglobinemia adquirida - más común que la congénita - es secundaria principalmente a consumo de sustancias con propiedades oxidantes que aceleran la formación de metahemoglobina superando las vías enzimáticas (Gregg \& Prchal, 2018).

Los nitritos son poderosos agentes oxidantes que alcanzan mayores niveles de metahemoglobina cuando son ingeridos en lugar de inhalados, pero esta última es la vía de consumo más frecuente, y la utilizada en este caso (Romanelli et al., 2004). Otras sustancias que pueden provocar metahemoglobinemia son dapsona -el fármaco causal más frecuente-, trimetoprim, sulfamidas, benzocaína, metoclopramida y anilina y sus derivados. Esto puede tener implicancias para el tratamiento, particularmente en población $\mathrm{VIH}+$ por la eventual combinación entre el nitrato de amilo y trimetropin o dapsona.

En individuos previamente sanos niveles de 10 a 20\% pueden asociarse a cianosis periférica y central sin otros síntomas asociados; niveles superiores al $30 \%$ producen fatiga, cefalea y disnea. Una metahemoglobinemia sobre el $50 \%$ produce sopor y arritmias, pudiendo ser letal sobre el 70\% ( Cortazzo \& Lichtman, 2014; Gregg \& Prchal, 2018). En individuos con reserva cardiopulmonar disminuida, sus efectos pueden ser mayores a niveles bajos.

Los principales hallazgos que deben hacer sospechar la metahemoglobinemia son el antecedente del consumo y la disociación entre las saturaciones de la oximetría de pulso y los gases arteriales, ambos presentes en el caso descrito. Por lo tanto, la disminución del porcentaje de oxigenación medido en la saturometría de pulso no es proporcional al nivel de metahemoglobina presente en la sangre del individuo. (Cortazzo \& Lichtman, 2014).

El manejo de la metahemoglobinemia comienza con la mantención de una vía aérea segura y oxigenoterapia, como se realizó en servicio de urgencias al ingreso del paciente presentado. Además, se debe suspender el agente causal. El tratamiento específico se recomienda para pacientes sintomáticos o con niveles de metahemoglobinemia sobre $20-30 \%$ según el riesgo individual (antecedente de anemia, insuficiencia cardiaca congestiva, enfermedad pulmonar obstructiva crónica). En general, cuando los valores de metahemoglobina sanguínea son menores al $10 \%$ los mecanismos naturales de reconversión pueden compensarlo sin necesidad de terapia activa (Wilkerson, 2010; Price, 2011).

El tratamiento específico más aceptado es el azul de metileno endovenoso en dosis de 1-2 mg/kg, el cual logra una respuesta dentro de la primera hora en la mayoría de los casos, siendo inhabitual requerir repetir la dosis por persistencia de niveles elevados (Modarai et al., 2002; Price, 2011; Al-Lawati \& Murch, 2012; Cortazzo $\&$ Lichtman, 2014). Actúa reduciendo el grupo férrico a través de la vía del NADPH. Se debe destacar que su uso está contraindicado en casos de deficiencia de glucosa-6-fosfato deshidrogrenasa ya que el efecto depende de su metabolismo y por el riesgo de hemólisis aguda secundaria a la acumulación del azul de metileno.

Una alternativa al uso de azul de metileno es el ácido ascórbico (vitamina C) por su efecto antioxidante, terapia finalmente utilizada en el caso presentado. No obstante, algunos autores no recomiendan su uso pues se requerirían altas dosis para superar la velocidad de reconversión fisiológica (Price, 2011) y por la presencia de efectos adversos en el manejo de metahemoglobinemia de otras etiologías (Lee \& Park 2014; Park et al., 2014; Rino et al., 2014).

Adicionalmente, en el contexto de intoxicación se debe descartar el consumo de otras sustancias; en este caso se complementó el estudio con Inmunoensayo automatizado / Cromatografía gaseosa con espectrometría de masa. Por otra parte, según la sospecha clínica y la disponibilidad del examen, se puede estudiar la deficiencia de glucosa-6-fosfato deshidrogenasa para descartar una causa subyacente y contar con el antecedente para definir el manejo ante un nuevo episodio. La búsqueda de causas congénitas se reserva para casos seleccionados, una vez descartadas las causas adquiridas.

\section{Tablas y figuras}

Tabla 1: Laboratorio Sanguíneo de ingreso:

\begin{tabular}{l|l|l|l}
\hline Parámetro & Resultado & Parámetro & Resultado \\
\hline Hemoglobina & 14,6 & $\mathrm{pH}$ & 7,41 \\
\hline Hematocrito & $41,5 \%$ & $\begin{array}{l}\mathrm{PaO} 2 \\
(\mathrm{FiO} 235 \%)\end{array}$ & 110 \\
\hline Plaquetas & 287.000 & PaCO2 & 31,2 \\
\hline Creatinina & 0,77 & Bicarbonato & 21 \\
\hline Sodio & 137 & Exceso de base & $-4,2$ \\
\hline Potasio & 3,6 & Ácido láctico & 2,9 \\
\hline Cloro & 100 & MetaHb* & $29,9 \%$ \\
\hline
\end{tabular}

*Metahemoglobinemia, valor normal 0,4 - 1,5\% 


\section{Conclusiones}

A nivel internacional se ha reportado que la mayoría de los pacientes que presentan una metahemoglobinemia por consumo recreacional de nitrito de amilo evolucionan favorablemente. El manejo consistirá en asegurar una vía aérea permeable y aportar oxígeno, reservando para casos seleccionados el azul de metileno y/o ácido ascórbico. Asimismo, se debe considerar la búsqueda de intoxicación concomitante por otras sustancias.

De acuerdo a nuestra revisión, este sería el primer caso de metahemoglobinemia secundaria al consumo de nitrito de amilo con fines recreativos descrito en Chile. Lo presentamos porque pronosticamos que el consumo de esta sustancia aumentará, tal como ha sucedido en otras regiones del mundo.

\section{Fuentes de financiamiento}

El trabajo no presenta fuentes de financiamiento

\section{Contribuciones y conflictos declarados por los autores}

Los autores no reportan conflictos de intereses.

\section{Referencias}

Al-Lawati A\& Murch N. Acquired methemoglobinaemia. (2012). Sultan Qaboos University Medical Journal 12, 237-41.

Bernasconi B, Konrad C\& Fischer S. (2014). [Reporte de caso - una intoxicación peligrosa después de la ingestión de nitrito de alquilo ("poppers")]. Anästhesiologie, Intensivmedizin, Notfallmedizin, Schmerztherapie: AINS (Alemán) 49, 654-658.

Cortazzo JA \& Lichtman AD. (2014). Methemoglobinemia: a review and recommendations for management. Journal of Cardiothoracic and Vascular Anesthesia 28, 1043-1047.

Gregg X \& Prchal J. (2018). Red blood cell enzymopathies. En Hematology: Basic Principles and Practice, ed. Hoffman R et al, pp 616-625. Elsevier.

Hunter L, Gordge L, Dargan PI \& Wood DM. (2011). Methaemoglobinaemia associated with the use of cocaine and volatile nitrites as recreational drugs: a review. British Journal of Clinical Pharmacology 72, 18-26.

Lee KW \& Park SY. (2014). High-dose vitamin C as treatment of methemoglobinemia. The American Journal of Emergency Medicine 32, 936.
Li D, Yang X, Zhang Z, Qi X, Ruan Y, Jia Y, Pan SW, Xiao D, Jennifer Huang Z, Luo F \& Hu Y. (2014). Nitrite inhalants use and HIV infection among men who have sex with men in China. BioMed Research International 2014, 365261.

Luis J, Virdi M, Nabili S. Poppers retinopathy. (2016). BMJ Case Reports 2016, bcr2016214442.

Malhotra R \& Hughes G. (1996). Methaemoglobinaemia presenting with status epilepticus. Journal of Accident \& Emergency Medicine 13, 427-430.

McCabe A, McCann B\& Kelly P. (2012). Pop goes the O2: a case of popper-induced methaemoglobinamia. BMJ Case Reports 2012, bcr2012007176.

Modarai B, Kapadia YK, Kerins M\& Terris J. (2002). Methylene blue: a treatment for severe methaemoglobinaemia secondary to misuse of amyl nitrite. Emergency medicine journal: EMJ 19, 270-271.

Pajarón M, Claver G, Nogué S \& Munné P. (2003). Metahemoglobinemia secundaria al consumo de poppers con ánimo recreativo. Medicina clínica 121, 358.

Park SY, Lee KW \& Kang TS. (2014). High-dose vitamin C management in dapsone-induced methemoglobinemia. The American Journal of Emergency Medicine 32, 684.e1-684.e3.

Pepper N, Zúñiga M \& Reed M. (2019). Prevalence and correlates of "popper" (amyl nitrite inhalant) use among HIV-positive Latinos living in the U.S.-Mexico border region. Journal of ethnicity in substance abuse [Pendiente publicación] [citado 14 de enero de 2020]. Disponible en: https://doi.org/10.1080/15332640.20 18.1540955

Price D P. (2011). Methemoglobin Inducers. En Goldfrank's Toxicologic Emergencies, ed Flomenbaum N, Goldfrank LR, pp 1698-1707. McGraw Hill Professional.

Rino PB, Scolnik D, Fustiñana A, Mitelpunkt A \& Glatstein M. (2014). Ascorbic acid for the treatment of methemoglobinemia: the experience of a large tertiary care pediatric hospital. American journal of therapeutics 21, 240-243.

Romanelli F, Smith KM, Thornton AC \& Pomeroy C. (2004). Poppers: epidemiology and clinical management of inhaled nitrite abuse. Pharmacotherapy 24, 69-78. 


\section{Muñoz et al.}

SENDA. (2017). Décimo segundo Estudio Nacional de Drogas en población escolar de Chile. Observatorio chileno de drogas del Servicio Nacional para la Prevención y Rehabilitación del Consumo de Drogas y Alcohol, SENDA, Ministerio del Interior y Seguridad Pública. Gobierno de Chile, pp 318. Accedido en https://www. senda.gob.cl/observatorio/estudios/poblacion-escolar/ el 14 de enero de 2020

SENDA. (2015). Décimo segundo Estudio Nacional de Drogas en población general de Chile. Observatorio chileno de drogas del Servicio Nacional para la Prevención y Rehabilitación del Consumo de Drogas y Alcohol, SENDA, Ministerio del Interior y Seguridad Pública. Gobierno de Chile, pp 17.]. Accedido en https://www. senda.gob.cl/observatorio/estudios/poblacion-general/ citado 14 de enero de 2020
Vaccher S, Hammoud M, Bourne A, Lea T, Haire B, Holt M, Saxton P, Mackie B, Badge J, Jin F, Maher L \& Prestage G. (2020). Prevalence, frequency, and motivations for alkyl nitrite use among gay, bisexual and other men who have sex with men in Australia. The International journal on drug policy 76, 102659.

Wilkerson RG. (2010). Getting the blues at a rock concert: a case of severe methaemoglobinaemia. Emergency Medicine Australasia 22, 466-469.

Wu LT \& Ringwalt CL. (2006). Inhalant use and disorders among adults in the United States. Drug and alcohol dependence 85, 1-11.

Wu LT, Schlenger WE \& Ringwalt CL. (2005). Use of nitrite inhalants ("poppers") among American youth. The Journal of adolescent health 37, 52-60. 